\title{
Design and Analysis of a Dynamic Scheduler for a Flexible Assembly System
}

\author{
Tz-Shian Huang, Li-Chen Fu, and Yung-Yu Chen \\ Dept. of Computer Science and Information Engineering \\ National Taiwan University, Taipei, Taiwan, R.O.C.
}

\begin{abstract}
This paper proposes a rule-based dynamic scheduler for a flexible assembly system. We first introduce a flexible control system developed by Intelligent Robot and Automation Laboratory in National Taiwan University. Based on that control system, the relationship between the control system and the scheduler is characterized. With focus on realization, hardware limitations such as long computation time and excessive memory-space usage are relaxed by incorporating several heuristic measures. The present work is applied to the simulated robotized flexible assembly system in our laboratory.
\end{abstract}

\section{Introduction}

In lieu of increasing complexity of assembly tasks and the desire for complete automation, integration of sensors, such as CCD cameras, force-torque sensor, optical switch and tactile sensor, into assembly workstations and employment of AI techniques for solving problems become the natural resort. Thus, for nowadays assembly process robots can become more intelligent and more capable of dealing with extremely difficult tasks. To date, there have been extensive research on applying knowledge-based approaches to the modeling of a robotic assembly cell, industrial applications, etc. $[1,2]$. Advantages of the above results include good problem solving techniques and nice system architecture, but disadvantage also exists in requiring expert engineers to extract knowledge from assembly jobs and assembly cell. The work by Huang and Lee [3] had also proposed a knowledge-based approach which assumes fixed assembly time. This resulting method with fixed assembly time assumption may be more suitable for the assembly tasks in a flow-line machine shop. For the sake of comparison, Noronha and Sarma [4] analyzed different knowledge-based approaches to solve the scheduling problems. Then, they presented a taxonomy of planning and scheduling and integrated the $\mathrm{AI}$ approaches to the knowledge rep :sentation. In this paper, we use a rule-based inference model to solve the scheduling problem in the flexible control system. According to the assembly AND/OR graph and the configuration of the multi-robot assem- bly system, the assembly rules can be generated systematically. In this paper, IDA* search algorithm is applied to implement the inference engine. In order to enhance the performance of the scheduler, several heuristics are adopted for the inference process. Futuremore, we propose and analyze several evaluation function for the flexible assembly system. Finally, we apply the flexible control system and the scheduling unit to a real experimental two-robot assembly cell. This paper consists of six sections. Section 1 is an introduction of the concept of a robotized assembly system and of assembly scheduling. Section 2 describes a general control system for this robotic assembly environment and its scheduling unit. The problems to be solved are also defined in this section. Section 3 introduces a rule-based inference engine and describe how can it be used to model the flexible assembly system. Section 4 describes how to implement the inference engine by incorporating IDA* search. An experiment is provided in Section 5 to demonstrate the flexibility and the enhanced performance of the inference engine. Finally, some conclusion are made in Section 6 .

\section{Robotized Flexible Assembly Sys- tem}

\subsection{System Overview}

A flexible assembly system (FAS) consists of a group of processing stations, interconnected by means of an automated material handling and storage system, and controlled by an integrated computer system. The major advantage of the FAS over a traditional assembly line is flexibility. Some critical piece of equipment should be taken into consideration when configuring an assembly workstation, which includes robot, part loader, transfer mechanism, assembly fixture, sensor system, computer control system.

\subsection{Flexible Control System - EDAK}

Jann [5] has proposed an object-oriented model for a control system architecture of robotic assembly automation; called event driven automation kernel (EDAK). There are four basic entities in the EDAK,

This research is sponsored by National Science Council, R.O.C., under the grant : NSC 85-2212-E-002-078. 
namely, Control Kernel, Application Task, Handler, Scheduler.

The system working scenario is that the eventdetection handler informs the control kernel that something just occurs, such as part's coming, assembly execution, etc., then the control kernel will update the system states and inform the system scheduler to make an optimal decision.

\subsection{Scheduler for the Flexible Control System}

EDAK provides a convenient and uniform environment for the scheduler for an FAS. The assembly system scheduling problem can be described as follows: Given the following data: 1) System configuration, 2) The product assembly knowledge, the objective of the scheduler is to decide the "best" action for the system. The "best action" may be different for different criteria. In this paper, we minimize the total makespan of the final product assembly, or somewhat equivalently, the throughput rate.

The scheduler is a special handler of the control kernel, whereby all operations and decision makings can be made.

\section{Rule-Based Modeling of Flexible As- sembly Systems}

\subsection{Rule-Based Inference System}

The logistic view of the scheduling process for an FAS is shown in Fig. 1. First, the modeling of an assembly system involves the transfer of the AND/OR graph and product assembly knowledge into the assembly rules for the assembly scheduling. Given the assembly rules and the state of the assembly system, the inference engine thus generates all of the possible assembly tasks, among which, its search algorithm will then search for the optimal ones.

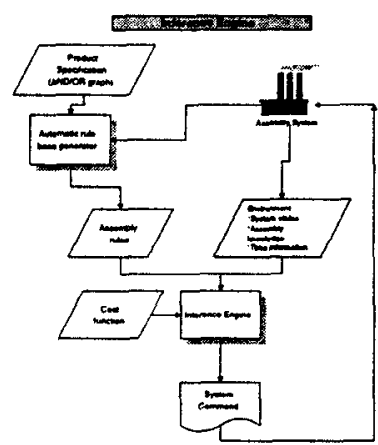

Figure 1: The hierarchical view of the assembly scheduling for an FAS.

\section{Definition 1 (State)}

A state is a predicate (or several predicates) which can describe a fact (or several facts). A state may has binding variable, i.e., a variable whose attribute is yet to be determined, and will appear only in a production rule (abbrev. rule). A state is called a condition if it is in the precondition part of some rule.

Definition 2 (Environment)

An environment represents the whole or partial system information, which contain the following information:

- State list: A list of states altogether represent the status of all resource usage and the knowledge related to assembly sequences.

- System time: The current time of the configuration to be registered.

- Delayed state list: This is a list of two-tuple pairs, $(T, S)$, where $T$ is the delay time and $S$ is the associated state, which means that the state $S$ will be added into the state list of the environment right after time interval $T$.

Definition 3 (Rule)

$A$ rule, $R$, is defined by a 7 -tuple, i.e., $R=(P, D, A$, Delay, Action, Priority, U), where

- $P$ : a set of states that describe the precondition of the rule.

- D: delete_list, a set of states that will be deleted from the system environment when the rule is executed.

- A: add_list, a set of states that will be added to the environment after the rule is executed.

- Delay: the execution time of the rule, which may be modeled as a constant or a stochastic variable (uniform distribution, Gaussian distribution or any other probabilistic distribution).

- Action: an external command that should be issued to the control system when the rule is fired

- Prioriry $\in[1, n]:$ the firing priority, where $n$ is the highest possible priority.

- U: unconditional rule, which will be fired in every constant or stochastic time interval.

Definition 4 (Inference Engine)

Given a set of rules and an environment, an inference engine is a mechanism which will try to generate the best actions (commands) for a given environment by the following steps:

1. The inference engine tries to find all enabled rules for the given environment. 


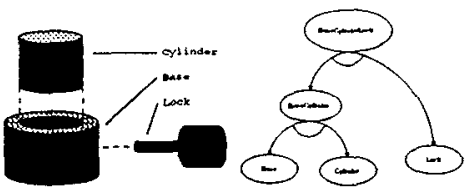

Figure 2: A simple product and its AND/OR representation.

2. If the environment fails to match any rule precondition, the inference engine update the environment in the time frame until some unconditional rules are enabled or the updated environment will match the preconditions of some rules.

3. If both of the above fail, the inference engine will stop; otherwise, it will choose the best rule from all the enabled rules for execution.

\section{Remarks:}

1. By updating the environment in the time frame, the inference engine will update the system time and remove the states that have the shortest delay time $T$ from the delayed state list and add those into the state list of the environment. Up to certain time instant, if this updated new environment enables any single rule, then the updating process will be interrupted by some state merging due to a rule firing.

2. To choose the best rule out of all the enabled rules for firing, the inference engine needs to analyze the system state from the environment according to the so-called evaluation model and decide which rule is the best for the environment. If that is done, the states in the delete-part of the chosen rule are removed from the state list of the environment.

\subsection{Modeling of a Robotized Flexible As- sembly System}

\subsubsection{Environment Representation}

The state representation contains two main pieces of information, namely, the general environmental information and the assembly task information. The first one includes the information to describe the assembly environment of a multi-robot assembly system, whereas the second one includes the information that expresses the desired product specification and the list of steps to complete the product assembly.

\subsubsection{Assembly Rule Formation}

In the following, we will demonstrate the formation of an assembly rule by using the product specification shown in Fig. 2. Note that this product requires
Table 1 The rules for $o p 1$ and op2 in the AND/OR graph in Fig. 2.

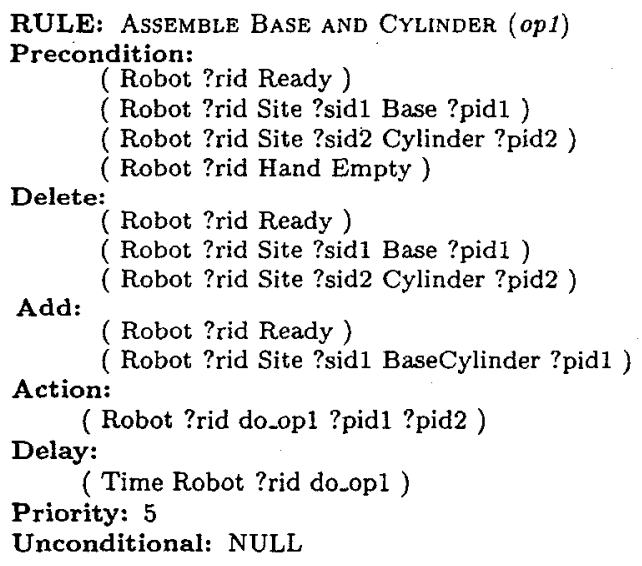

two types of assembly commands, op1 and op2. The AND/OR graph, indicates that op1 is the operation which assembles Cylinder and Base into the subassembly BaseCylinder. To execute this operation, a free robot is needed first and then both of the aforementioned parts are ready for assembly.

After the operation is started, we must delete some states to indicate that it is now in progress.

After the operation is done, the robot will produce one subassembly, BaseCylinder, on site ?sid1 and the robot, ?rid, will become ready again. The complete rules will be shown in Table 1 .

\section{Search-Based Dynamic Scheduler}

\subsection{Search-Based Assembly Dynamic Scheduling}

The objective of the inference engine is equivalent to finding the best rule for the current environment. The function of the inference engine can be decomposed into three, one is to find the enabled rules, another is to choose the best rule from these enabled ones, and the other is to fire the rule. In the following, we describe how to apply search algorithm to the above problem.

\subsubsection{IDA* Search Algorithm}

The searching strategy in this paper is based on iterative-deepening $A^{*}, I D A^{*}$ for short, search algorithm [6]. Iterative-deepening reduces the space complexity to linear while preserving optimality. With an admissible heuristic estimate function (i.e., one that never overestimates), IDA* is guaranteed to find the optimal solution path. Moreover, it has been proved that IDA* obeys the same asymptotic branching factor as $\mathrm{A}^{*}$, if the number of nodes grows exponentially with the solution depth [6]. This growth rate is called the 
heuristic branching factor $b_{h}$. The better space effciency of $\mathrm{IDA}^{*}$ is paid for an increased number of node expansions. On the average, $\mathrm{IDA}^{*}$ requires $b_{h} /\left(b_{h}-1\right)$ times as many operations as $A^{*}[7]$.

\subsubsection{Matching Algorithm}

Given an environment, the matching algorithm will find all enabled rules and the corresponding binding table for the rule from the rule database. Algorithm 1 and 2 below show the prototype of the rule-matching.

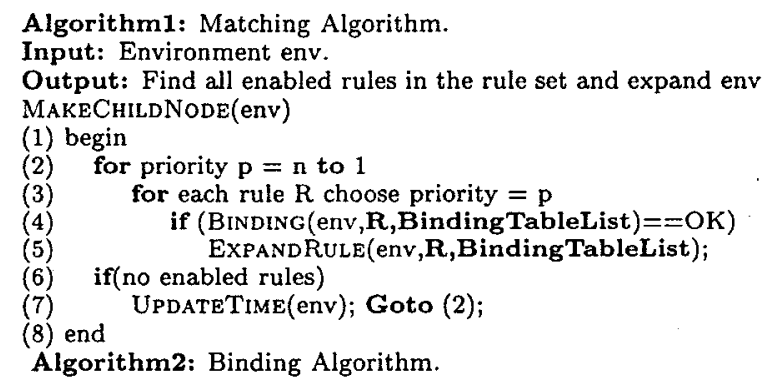

Input: Environment env, rule $\mathbf{R}$.

Ouput: Find all binding tables and store them into BindingTableList.

BINDING(env,R,BindingTableList)

(1) begin

(2) for each Binding Table in the BindingTableList

(3) for each precondition $p$ of rule $R$

(4) Apply Binding Table to $p$.

(5) if ( $p$ matches some state $s$ in the state list of env)

(6) Resolve binding variable from $p$ and then store it in Binding Table.

(7) if $p$ matches more than one state in the env

(8) Resolve binding variable and add a new Binding Table in the list.

(9) else return FAIL

(10) return OK

(11) end

\subsection{Improve The Search Algorithm}

Because IDA* algorithm does not retain path information from one iteration to the next, the shallow tree parts are reexamined several times. There are several methods that improve the search performance by gathering information in the process of iterative-deepening. Here, we examine two type of strategies, node ordering heuristics and avoid re-expansion heuristics.

\subsubsection{Node Ordering Heuristics}

SORT : This heuristic is based on rearrangement of the successors $n_{i}$ of the interior node $n$ in decreasing order of their heuristic estimates $h\left(n_{i}\right)$. Successors with high estimates, which means the node are closer to the goal, are visited first. Although this scheme helps one in his search for optimal solutions, the savings achieved in (optimal) IDA* search rarely compensate for the increased overhead $[8$, p. 471].

\subsubsection{Avoid Re-Expansion Heuristics}

Some researches discuss whether it is better to use graphs than to use trees $[9,10]$. In such cases, memory functions should be employed to avoid unnecessary reexpansions of previously visited nodes [11].

CYCLE : A moving cycle is a sequence of operators, which after going through some intermediate states from the starting state, finally returns to the starting state. Since iterative deepening maintains the current search path, moving cycles can be eliminated by comparing each newly generated node with those on the current path and pruning node that already appears on the path.

TRANS : Move transpositions arise when different paths end in the same node. They can be traced with a transposition table which stores every visited node and the cost bound at which the position has been searched. When the current node is found in the table, its subtree can be pruned if the remaining cost bound is less or equal to the corresponding bound retrieved from the table. A good hashing function is required and must be designed case by case. The size of the transposition table should be allocated as large as possible. Reinefeld and Marsland have shown that this heuristic can reduce the search node visited by $\mathrm{IDA}^{*}$ in [12].

\subsection{Evaluation Function for Flexible As- sembly System}

Many off-line scheduling problems usually assume that the system is deterministic and the performance measure is the total makespan. However, for an on-line scheduler, to minimize the total makespan is difficult. In this paper, the objective of the scheduler is to minimize the production time for every product assembly, or, somewhat equivalently, the throughput rate.

We first define productive operation and nonproductive operation. The definition of productive operation is the operation which will change a part or produce a new part. In an assembly system, "ASSEMBLE", "SCREW" are all productive operations, whereas "MOVE", "PICK" and "PUT" are nonproductive operations.

Now we define $g(e n v)$ as the time spent from the root environment to the current environment, and $h(e n v)$ as the estimated time required to produce some final product.

For every final product assembly, find the estimated time to finish the assembly of the product. The value of $h(e n v)$ will be the smallest value of the estimated time.

STEP 1 : For every product, find all parts required for it.

STEP 2 : Perceive that these parts in Step 1 are all sitting in the assembly sites of an available robot, 
and then calculate the total time required to finish the assembly of the product. Because all parts are in the robot sites, all operations required are productive ones. We call this time period as productive operation time, $T_{p}$.

STEP 3 : In practice, there are usually some parts required for the final product which are currently not ready yet. They can be either fed from the part loading machine or obtained from the buffer. Thus, for any part which is not yet in the robot site, we add one estimated operation time, $T_{\text {estimate }}$, which is the smallest time elapse for the robot to get the part from buffer, conveyor belt, or other sites. If there are $n$ parts required to finish the product which are not currently ready in the robot sites, then the total nonproductive operation time, $T_{n p}=n \cdot T_{\text {estimate }}$.

For example, consider a simple assembly cell which contains only one robot. This cell is to produce the product, denoted as part5, whose AND/OR assembly graph is shown in Figure 3. Assume now the robot's site contains one part, part3. In this example, the robot is unable to produce part5 because of lack of part 4. Assume that the next part loading will load part4 into the cell, which normally takes 5 seconds. Further, assume the operation time is 12 seconds for the robot. Thus, $h(e n d)=5+12=17$ seconds and $f(e n v)=10+17=27$ seconds.

Theorem 1 The evaluation function, $h(e n v)$, defined above is admissible.

Proof:

According to the above definition, the estimated time to the goal for a given environment is as follows:

$$
h(e n v)=\operatorname{Min}\left(T_{p_{i}}+T_{n p_{i}} \mid \text { For every product } i\right)
$$

Comparing $h(e n v)$ with the real operation time $T^{*}$ required to reach the goal for the given $e n v$, we can find that $T_{p}=T_{p}^{*}$ and $T_{n p} \leq T_{n p}^{*}$, where $T_{p}^{*}$ and $T_{n p}^{*}$ represent the real productive operation time and the real nonproductive operation time required to goal respectively. Thus, the estimate function, $h(e n v)$ is admissible.

A cost function is said to be monotonic if the cost of a child is always greater than or equal to that of its parent. Korf prove that, with a monotonic cost function, iterative-deepening search expands nodes in the best-first order [13, p.45].

Theorem 2 The cost function, $f(e n v)$, defined above is monotonic.

The proof is shown in appendix.

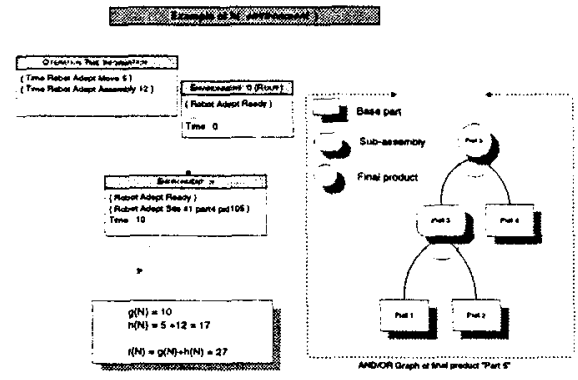

Figure 3: An example of the estimate function, $h(e n v)$.

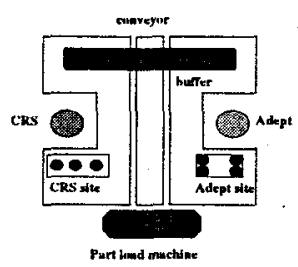

Figure 4: System layout

\section{Experiment and Analysis}

We are going to demonstrate an example solved by the proposed inference engine and dynamic scheduler. The example is the scheduling problem for an experimental robotized flexible assembly system in our laboratory.

The system is two-robot assembly cell which is dedicated to assembling various types of mechanical parts serially sent in through a conveyor belt.

All the parts are sent into the cell through a conveyor belt in a serial order, and are on-line identified using an overhead camera and a side camera. Both robots are identical in terms of their assembly function, i.e., each of them can perform any type of assembly task. Buffers are assumed to be present in order to store the parts which already arrived but are not yet ready to be assembled. The whole system is controlled by EDAK. The equipment structure is shown in Fig. 4.

\subsection{Experimental Result}

The experiment is a multi-robot simulation based on the software environment called CimStation runs on a Sun-Sparc 10 workstation. The algorithm of each function unit in simulation is written in SIL which is a robotic simulation language, while the assembly scheduling programs are written by $\mathrm{C}++$ language. In the simulation, both robots have four assembly sites. The average time for generating each schedule is about 2-10 seconds.

The average number and maximum of buffer usage are 6.322 and 10 respectively. The utilization of each robot, Adept and CRS, are shown in Figure 5 . 


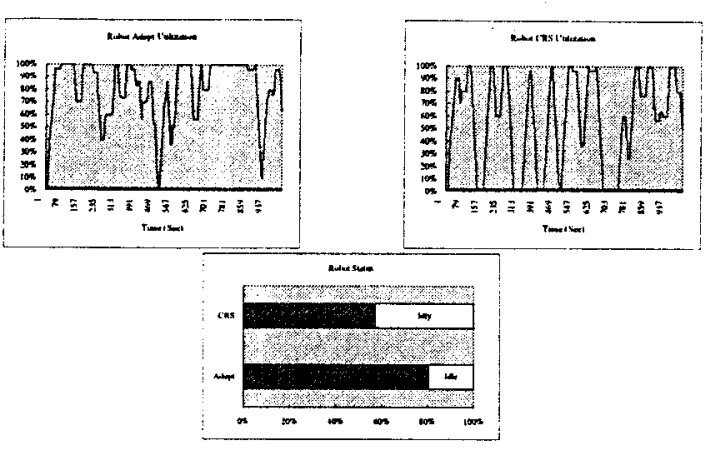

Figure 5: Utilization of robot Adept and CRS.

\section{Conclusion}

This paper is an extension of [14]. The relationship between the scheduler and the EDAK is discussed in Section 2.

The result of the improved IDA* algorithm in Section 4.2 is consistent with the research by Reinefeld [12]. It is noteworthy that the most time consuming part is the matching procedure. The improved matching procedure in Section 4.2 is similar to RETE [15]. Efficiency arises from its exploitation of temporal redundancy in working memory and structural similarity among productions.

\section{Appendix}

The cost function, $f(e n v)$, defined in section 4.3 is monotonic. Proof:

For all nodes $n^{\prime}$ and $n$, where $n^{\prime}$ is a child of $n$,

$$
\begin{gathered}
f(n)=g(n)+h(n) \\
f\left(n^{\prime}\right)=g\left(n^{\prime}\right)+h\left(n^{\prime}\right)
\end{gathered}
$$

The firing rule between node $n$ and $n^{\prime}$ may be one of the following kind of rule.

1. Control rule: No actual command is issued. In this case, the environment time will not change. Thus, $g(n)=g\left(n^{\prime}\right)$ and $h(n)=h\left(n^{\prime}\right)$. So that, $f(n)=f\left(n^{\prime}\right)$.

2. Productive operation: If the operation takes time $T_{o p}$, then $g\left(n^{\prime}\right)=g(n)+T_{o p}$ and $h\left(n^{\prime}\right)=$ $h(n)-T_{o p}$. Thus, $f\left(n^{\prime}\right)=f(n)$.

3. Nonproductive operation: Assume the operation takes time $T_{o p}$. Thus, $g\left(n^{\prime}\right)=g(n)+T_{o p}$, but there are two possible cases for the estimate function:

(a) If this operation takes one primitive part required for the product assembly, the estimated time to reach the goal will be decreased by $T_{\text {estimate, }}$, i.e., $h\left(n^{\prime}\right)=h(n)-T_{\text {estimate }}$, because one new part is now in the robot site. Because $T_{\text {estimate }} \leq T_{\text {op }}$, we have $f\left(n^{\prime}\right) \geq f(n)$.

(b) Otherwise, $h\left(n^{\prime}\right) \geq h(n)$, and thus $f\left(n^{\prime}\right) \geq$ $f(n)$.

Consequently, the cost function, $f(e n v)$, is monotonic.

\section{References}

[1] M. S. Fox and S. F. Smith, "ISIS - a knowledge-based system for factory scheduling," Expert System, vol. 1, no. 1, pp. 22-49, 1984.

[2] H. V. Brussel, F. Cottrez, and P. Valckenaers, "SESFAC: a scheduling expert system for flexible assembly cells," in Proceedings of 1990 IEEE conference on Robotics and Automation, (Cincinnati, Ohio), pp. 1950-1955, 1990.

[3] Y. F. Huang and C. S. G. Lee, "A frame work of knowledge-based assembly planning," Proceedings of 1991 IEEE conference on Robotics and Automation, vol. 2, pp. 599-604, Apr. 1991.

[4] S. J. Noronha and V. V. S. Sarma, "Knowledgebased approaches for scheduling problems: a survey," IEEE Transaction on Knowledge and Data Engineer. ing, vol. 3, pp. 160-171, June 1991.

[5] C.-S. Jann, "Flexible control system for robot assembly automation," Master's thesis, National Taiwan University, Department of Computer Science and Information Engineering, 1994.

[6] R. E. Korf, "Depth-first iterative-deepening: An optimal admissible tree search," Artificial Intelligence, vol. 27, no. 1, pp. 97-109, 1985.

[7] M. E. Stickel and W. M. Tyson, "An analysis of consecutively depth-first search with applications in automated deduction," in Proceeding 9th International Joint Conference on Artificial Intelligence, pp. 1073$1075,1985$.

[8] C. Powley and R. E. Korf, "Single-agent parallel window search," IEEE Transaction on Pattern Analysis and Machine Intelligence, vol. 13, pp. 466-477, May 1991.

[9] R. Ramaswamy and A. K. Sen, "Single machine scheduling as a graph search problem with pathdependent arc costs," in Proceedings ECAI-92, Europ. Conference Artificial Intelligence, (Vienna), pp. 1-5, Aug. 1992.

[10] A. Mahanti, S. Ghosh, D. S. Nau, A. K. Pal, and L. Kanal, "Performance of IDA* on trees and graphs," in Proceeding 10th National Conference on Artificial Intelligence, AAAI-92, pp. 539-544, 1992.

[11] J. C. Pemberton and R. E. Korf, "Incremental path planning on graphs with cycles," in Proceedings of the 1st International Conference on Artificial Intelligence Planning Systems, (College Park, MD, USA), pp. 179188, Morgan Kaufmann Publ Inc, June 1992.

[12] A. Reinefeld and T. A. Marsland, "Enhanced iterative-deepening search," IEEE Transaction on Pattern Analysis and Machine Intelligence, vol. 16, pp. 701-710, July 1994.

[13] R. E. Korf, "Linear-space best-first search," Artificial Intelligence, vol. 62 , pp. 41-78, 1993.

[14] H.-H. Hsu, "Fully automated robot assembly cell: scheduling and simulation," Master's thesis, National Taiwan University, Department of Computer Science and Information Engineering, 1994.

[15] C. L. Forgy and S. J. Shepard, "Rete, a fast match algorithm," AI Expert, pp. 34-40, Jan. 1987. 